\title{
Prevention of Entero-Atmospheric Fistula: The Initial Closure of the Abdomen
}

\author{
Voros $D^{3}$, Anastasopoulos $G^{3}$, Sarris $G^{3}$, Fotopoulos $A^{2}$, Marinis $A^{4}$, Dougli $K^{1}$
}

\begin{abstract}
Background and Aims: The "Open Abdomen" technique for difficult conditions such as trauma, necrotizing pancreatitis, severe peritonitis, reoperations and transplantations have become a very useful way to save patients. The more severe complication of this method are the enteroatmospheric fistulae with a frequency of 5-75\% and a mortality up to $42 \%$. So any effort to prevent these is very essential. The aim of this paper is to present methods of the initial (temporary) closure of the abdomen for the prevention of this catastrophe, when early approximation of the abdominal wall is not feasible.

Methods and Materials: We present two representative case reports and we describe the use of full thickness or split skin grafts as first step for abdominal closure.

Results and Conclusions: The outcome of our patients by using tissues for temporary abdominal closure seems to have fewer problems than other methods with synthetic materials. We think that this is a reasonable option for the cases where we cannot achieve approximation of the abdominal wall edges in the early days of an open abdomen.
\end{abstract}

Key words: Abdominal Compartment Syndrome; open abdomen; entero-atmospheric fistulae; closure of open abdomen; skin grafts in open abdomen; meshes in open abdomen

\section{Introduction}

The "open abdomen" technique during the last decades, has contributed largely in the survival of many thousands of patients with severe abdominal problems such as multiple trauma, severe peritonitis, necrotising pancreatitis, transplantations and reoperations.

The more dreadful complication of the "open abdomen" is the so called entero-atmospheric fistula or "open air fistula". The frequency of this complication is related to the duration that abdomen remains open and the different methods used for temporary coverage of the abdominal viscera, ranging from $5-75 \%[3,4]$. In cases using polypropylene mesh the frequency is increased to $12-50 \%$ [5]. The mortality of these fistulas in recent studies is up to $42 \%$ [3].

\footnotetext{
${ }^{1}$ Department Plastic Surgery, General Hospital of Athens, Greece

${ }^{2} 1^{\text {st }}$ Department of Surgery, Sismanoglion Hospital of Athens, Greece

${ }^{3} 3^{\text {rd }}$ Department of Surgery Henry Dunant Hospital Center, Athens, Greece

${ }^{4} 1^{\text {st }}$ Department of Surgery, Tzaneion Hospital of Piraeus, Greece

Corresponding author: Voros Dionysios

Emeritus Professor of Surgery, University of Athens

Chief of 3rd Department of Surgery, Henry Dunant Hospital Center

107 Mesogion Avenue, Athens 11526, Greece

Tel.+30-210 6972957, +30-6944561888

e-mail: diovoros@med.uoa.gr
}

Received Aug 26, 2020; Accepted Sep 2, 2020
As the consequences of fistulization is so severe, any effort to prevent it is very essential. Sometimes the abdomen is open for 2-3 weeks because the underlying problems do not permit a staged closure of the laparostomy, increasing the risk for fistula.

We present two recent cases, where we did the temporary (initial) closure of the open wound using biologic material (tissues).

\section{Case 1}

A 38-year-old male patient was referred to our hospital after six weeks of conservative treatment for necrotising pancreatitis following ERCP, sphincterotomy and stenting of the bile duct for stone disease. Cholecystectomy was performed 3 weeks before the ERCP. We decided to operate him on the next day. In the following month, we re-operated on him six times for necrosectomies, severe bleeding from transverse mesecolon (2 times), partial excision of transverse colon and terminal ileostomy.

He remained 25 days intubated for respiratory support and 30 days with open abdomen. One month after the first necrosectomy, we used for closure of the abdomen bilateral flaps of skin and all the subcutaneous fat leaving open the myo-fascial-edges except the suprapubic area (Figure 1). Six months, later we closed the ileostomy and reconstructed the transverse colon. Figures 2 and 3 show 


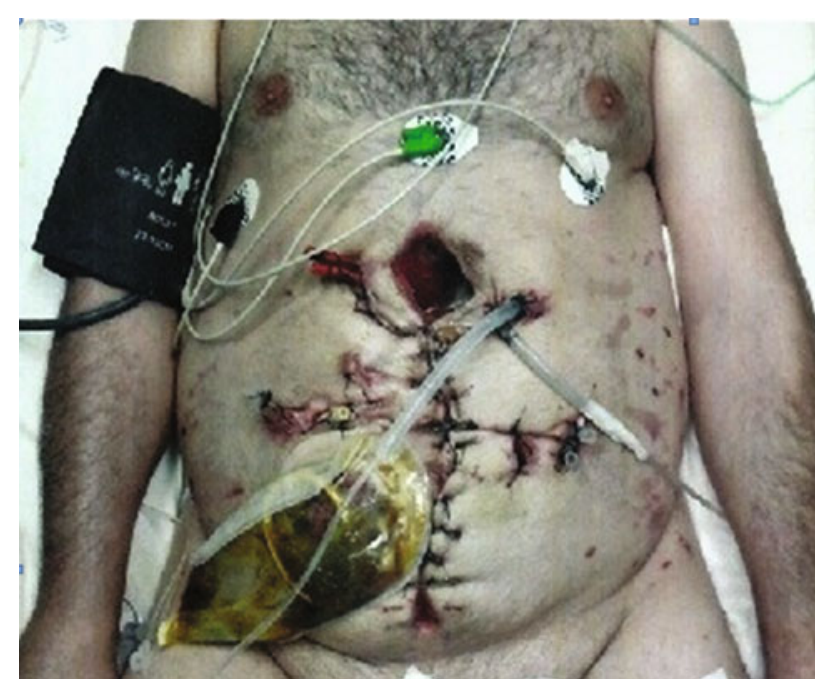

Figure 1. The initial closure of the abdomen of patient (Case 1).

the abdomen of the patient 14 months after the last operation. He is working normally.

\section{Case 2}

A 28-year-old male patient had a severe motorcycle accident with rupture of the descending aorta after blunt, chest and abdominal trauma. He was transferred to the nearest hospital and percutaneous stenting of the aorta was successfully performed. After this life saving procedure he was further submitted to multiple procedures for: rupture of the jejunum, delayed rupture of the ascending colon, leading to a loop colostomy and narcotizing soft tissue infections (NSTI)

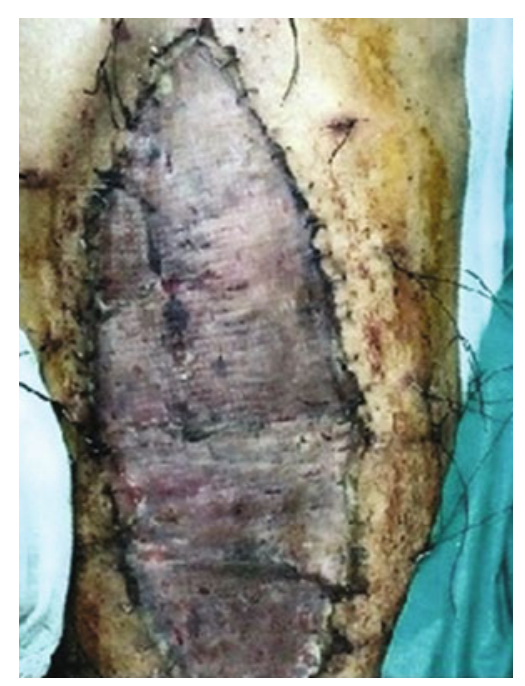

Figure 3. The initial, partial thickness skin coverage of the viscera in patient (Case 2).

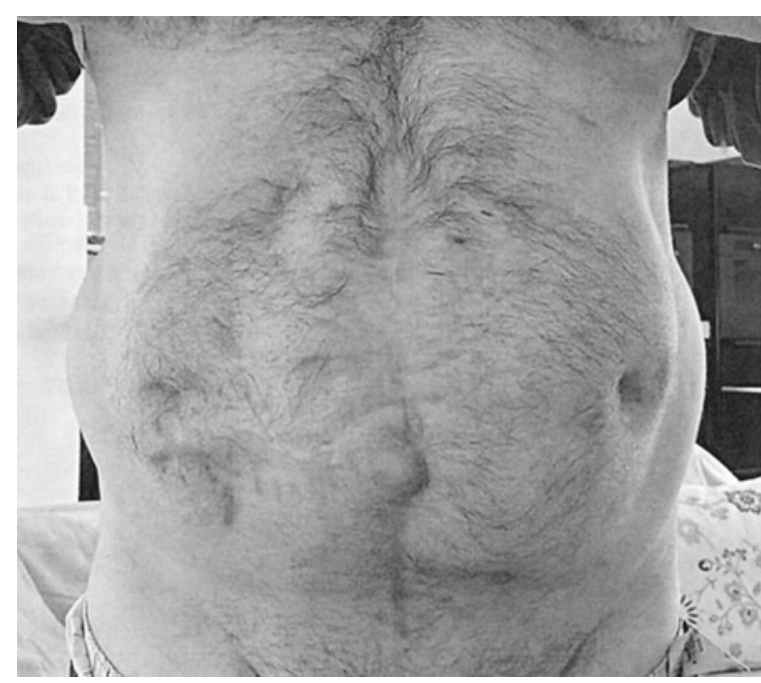

Figure 2. Picture of the abdomen 1 year later (Case 1).

After been in ICU with open abdomen for almost a month, we decided to apply the method of absorbable mesh (vicryl) followed by partial thickness (split) skin as temporary cover of the viscera (initial abdominal closure). The vicryl mesh was almost resolved leaving in place a soft layer of granulosous tissue on top of viscera that it was covered by skin graft taken from the anterior thigh (Figure 3). He had an uneventful recovery and ten days later he left the hospital.

Five months later (January 2020) we closed the colostomy (reconstruction of the right colon by end-to-end anastomosis).

The patient, as it was expected, developed a large ventral hernia (Figure 4) but has returned to work using an abdominal belt. After the COVID-19 pandemic has subsides, we plan to finally reconstruct the ventral hernia by using a double-layered non absorbable mesh.
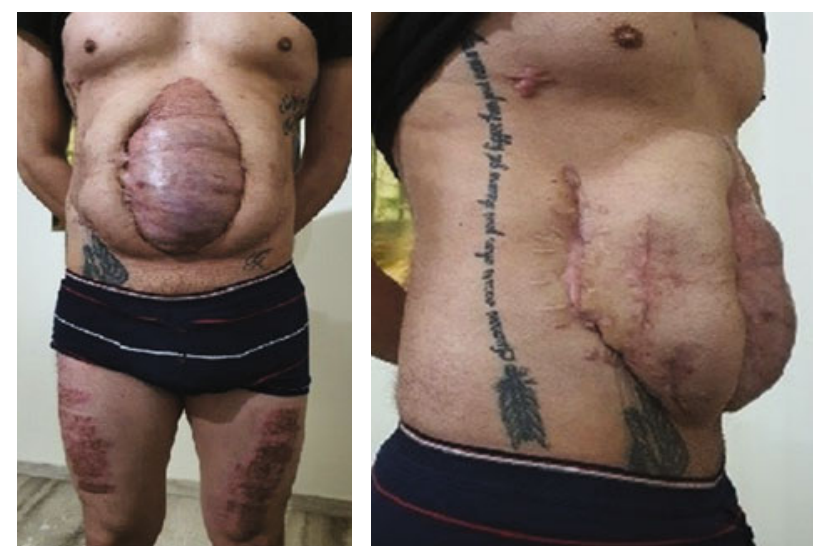

Figure 4. a, b Picture of the abdomen 8 months later (Case 2). 


\section{Concluding Remarks}

Although management of an enteroatmospheric fistula can be challenging $[3,6,7]$, methods to prevent its development are always welcome and interesting.

When we leave an abdomen open because the underlying conditions do not permit staged closure, it is reasonable and safe as initial coverage of the abdomen to choose either vascularised tissue (skin flaps) or absorbable mesh (vicryl) as an alternative for split-skin grafts.

Informed Consent: The authors declare that the study has been performed in accordance with the ethical standards laid down in the 1964 Declaration of Helsinki. Also all patients gave their written informed consent prior to their inclusion to the study.

Conflict of interest: The authors declare that there is no conflict of interest.

\section{References}

1. Ivutury RR, Drebel L, Porter JM, et al. Intira-abdominal
Hypertension and the abdominal compartment syndrome. Surg Clinic North Am 1977;77:783-800.

2. Lepaniemi A, Kirkpatrick A, Salazar A, et al. Miscellaneous Conditions and Intra-abdominal Hypertension. In: Ivatury R, Cleatham M, Malbrain M, Sugure M. Textbook Abdominal Compartment Syndrome. Medical Intelligence Unit: Landes Bioscience; c2006. p.197-216.

3. Latif Rifut. Beyond Damage Control Surgery: Abdominal Wall Reconstruction and complex Hernia Repair. In: Velmahos G, Degiannis E, Doll D, editors. Penetrating Trauma. 2nd Ed. Springer: Berlin, Heidelberg; c2017. p. 341-349.

4. Cambeli A, Chang M, Fabian T, et al. Management of the Open Abdomen: From initial operation to definite closure. American Surgeon 2009;75(11 Suppl):S1:p.22-23.

5. Goettler C, Rotondo M, Schwab C. Surgical management of the open abdomen after damage control or abdominal compartment syndrome. In: Ivatury R, Cheathan M, Mann $\mathrm{L}$, et al. Abdominal Compartment Syndrome. Landes Bioscience; c2006. p. 271-282.

6. Marinis A, Giokas G, Argyra E, et al. Enteroatmospheric Fistula': Gastrointestinal openings in the open abdomen: A review and recent Proposal of a Surgical Technique. Scandinavian Journal of Surgery 2013;102:61-8.

7. Demetriades D. A Technique of Surgical closure of complex intestinal fistulae in the Open Abdomen. Journal of Trauma 2003;55:999-1001. 